\title{
Sistema de oscilometria de impulso em pediatriar revisão de literatura
}

\section{Impulse Oscillometry System in pediatrics: review}

\author{
Maíra S. de Assumpção ${ }^{1}$, Renata M. Gonçalves ${ }^{1}$, Letícia G. Ferreira², Camila I. S. Schivinski ${ }^{3}$
}

\begin{abstract}
RESUMO
Introdução: a oscilometria de impulso (IOS) é considerado um método de avaliação da mecânica respiratória, e por ser não invasivo, vem sendo crescentemente indicado na assistência e investigação do paciente pediátrico. Estudos tem tido como interesse a determinação de valores de referência e a verificação da relação do IOS com outros métodos de avaliação respiratória. Objetivo: apresentar uma atualização da literatura quanto ao sistema, critérios técnicos e interpretação de dados, bem como, a relação do sistema com outros métodos de avaliação respiratória e sua aplicabilidade clínica. Método: revisão bibliográfica com consulta nas bases de dados MEDLINE/PubMed e Ebsco, utilizando as palavras-chaves: "impulse oscillometry", "reference values" e "child". Considerou-se todo material com informações sobre o equipamento, suas indicações e aplicação como instrumento de avaliação em diferentes situações clínicas. Resultados: foram identificados 45 documentos, sendo 41 ensaios clínicos e 4 revisões, complementados com 20 trabalhos: 1 guideline, 1 manual sobre o equipamento e 18 artigos referentes ao tema. Esse material foi caracterizado por abordar o equipamento, seus parâmetros de avaliação, técnica de execução e posicionamento do paciente durante o exame, critérios de aceitabilidade e reprodutibilidade e interpretação dos dados obtidos. São várias as publicações dessa ferramenta na avaliação da mecânica respiratória de disfunções como asma e fibrose cística e geração de valores de referência em crianças saudáveis. Conclusão: o IOS é um recurso relativamente novo, cuja indicação em pediatria tem sido crescente, mas que ainda carece investigações, tanto técnicas quanto clínicas.
\end{abstract}

Palavras-chaves: Oscilometria. Valores de Referência. Criança. Testes de Função Respiratória.

\section{Introdução}

O sistema de oscilometria de impulso- Impulse Oscillometry System (IOS) é um método oscilométrico para medida das propriedades mecânicas do pulmão e tórax, introduzida como uma modalidade alternativa para o teste de função pulmonar. Foi desenvolvido em 1956 por Dubois et al., a partir da teoria das vibrações, na qual, os impulsos são capazes de gerar oscilações de fluxo que se sobrepõem à ventilação espontânea permitindo desta forma analisar as respectivas respostas de variação de pressão e débito pulmonares., ${ }^{1,2,3}$
1. Fisioterapeuta, Mestranda do programa de Pós-graduação em Fisioterapia. Departamento de Fisioterapia da Universidade do Estado de Santa Catarina (UDESC), Florianópolis-SC/ Brasil.

2. Fisioterapeuta. Departamento de Fisioterapia / UDESC.

3. Fisioterapeuta, Doutora em Saúde da Criança e do Adolescente pela Universidade Estadual de Campinas (UNICAMP), Professora efetiva do curso de graduação e pós-graduação em Fisioterapia da UDESC, Florianópolis-SC/ Brasil.
Correspondência: Universidade do Estado de Santa Catarina (UDESC). Centro de Ciências da Saúde e do Esporte (CEFID). Rua Pascoal Simone, 358- Coqueiros. CEP: 88080-350. Florianópolis- SC.

Artigo recebido em 15/08/2013 Aprovado para publicação em 20/02/2014 
Surgiu como uma modificação da técnica de oscilações forçadas (TOF) ${ }^{4}$ e difere dessa basicamente por obter medidas de resistência e reatância em faixas de múltiplas frequências. ${ }^{5}$ Tal característica permite uma análise útil da variação na impedância respiratória. ${ }^{6}$

O IOS oferece as mesmas vantagens da TOF, como exigir o mínimo de requisitos para a cooperação do paciente, medições rápidas, fáceis e reprodutíveis. ${ }^{7,2} \mathrm{E}$, ao contrário das técnicas convencionais, como a espirometria, trata-se de um método que não utiliza os músculos respiratórios como fonte de força e sim um gerador externo de impulsos (alto-falante). ${ }^{1,2,8}$ Dessa forma, é um sistema que parece ser mais representativo em situações da vida diária, pois sua interpretação se dá pela respiração corrente, ao invés de manobras expiratórias forçadas.

Também é considerado uma ferramenta eficaz para avaliar o valor da resistência das vias aéreas ao longo da árvore traqueobrônquica (desde a zona central até a periferia) $)^{1,9,10}$ e desempenha importante papel na análise complementar à espirometria em pacientes com asma ${ }^{11-18}$, fibrose cística ${ }^{19,20,21}$ e rinite. $^{22}$

Em relação aos conceitos importantes desse sistema de avaliação da mecânica respiratória, as principais mensurações realizadas englobam a impedância $(Z)$, a resistência $(R)$, a reatância $(X)$ e a frequência de ressonância (Fres). A Z é expressa pelo cálculo da força total necessária para propagar uma onda de pressão através do sistema pulmonar ${ }^{23}$ e tem por definição ser a função de transferência, ou taxa de pressão ou fluxo que é derivada das oscilações forçadas sobrepostas $^{6}$, representa a carga mecânica total oferecida pelo sistema respiratório. ${ }^{24}$ Outro componente avaliado é a $R$, que corresponde à energia necessária para se propagar uma onda de pressão através das vias respiratórias, inclui a passagem por meio dos brônquios e bronquíolos e distensão do parênquima pulmonar $^{23}$, é proporcional ao fluxo e corresponde à parte "real", representando o coeficiente de viscosidade de um fluxo laminar, fazendo uma analogia com a eletricidade, corresponde à "resistência ôhmica", ou seja, o componente de energia que é convertido em luz por uma lâmpada. ${ }^{25}$ Já a $X$ é a energia gerada pela retração pulmonar, após a distensão por uma onda de pressão ${ }^{23}$, condiz às transformações de energia decorrentes da expansão do volume e da aceleração das massas. Esse componente retrata basicamente o acúmulo transitório e posterior do retorno de energia, inclui a força para mover a coluna de ar nas vias aére- as condutivas e as propriedades elásticas periféricas. ${ }^{25}$ E por fim, a Fres, que traduz a frequência com que o tecido pulmonar se move da distensão passiva para extensão ativa em resposta à força do sinal de onda de pressão. ${ }^{23}$ As curvas de impedância do IOS, que mostram a relação fluxo-pressão também podem ser correlacionadas ${ }^{26}$, equivalentes com a resistência e a inertância mecânicas em conformidade com o sistema respiratório humano e que podem ser representadas por modelos de circuitos elétricos. ${ }^{27,28,29}$

Nesse contexto, e considerando a importância da avaliação da mecânica respiratória na prática clínica, a presente revisão tem como objetivo apresentar uma atualização da literatura quanto ao sistema, critérios técnicos e interpretação de dados, bem como a relação do sistema com outros métodos de avaliação respiratória e sua aplicabilidade clínica.

\section{Fonte dos dados}

Foram consultadas as bases de dados para a revisão de literatura: MEDLINE/PubMed e Ebsco. As palavras-chaves utilizadas para a busca dos artigos foram: "impulse oscillometry", "reference values", "child". Não foram limitados idiomas e ano de publicação. A busca dos estudos foi realizada nos período de abril de 2011 a dezembro de 2013. Como critérios de inclusão do material na corrente revisão, se estabeleceu que o mesmo deveria abordar questões sobre o equipamento, suas indicações, aspectos técnicos, interpretação de dados, aplicação do sistema como instrumento de avaliação em diferentes situações clínicas e faixas etárias e a relação do sistema com outros métodos de avaliação respiratória ou associação com desordens respiratórias.

\section{Resultados}

Foram selecionados 45 documentos referentes ao tema abordado, sendo 41 ensaios clínicos e quatro revisões. Para complementação deste material foram considerados 20 trabalhos que não estavam indexados nas bases pesquisadas, sendo um guideline, um manual sobre o equipamento e 18 artigos referentes ao tema. Esse material foi caracterizado por abordar a aplicação do sistema principalmente na população pediátrica com disfunções respiratórias, como a asma e a fibrose cística. Também foram encontradas publicações referentes à geração de valores de referência em pediatria na população saudável. Com base nesses 
trabalhos, a síntese dos dados foi organizada em tópicos: 1) equipamento de oscilometria de impulso, 2) parâmetros de avaliação, 3) posicionamento e técnica de execução, 4) critérios de aceitabilidade e reprodutibilidade, 5) interpretação dos dados, 6) relação entre o IOS e outros métodos de avaliação do sistema respiratório, 7) IOS em pediatria e 8) estudos sobre valores de referência para faixa etária pediátrica.

\section{Síntese dos dados}

\section{Equipamento de oscilometria de impulso}

O equipamento é composto por um pneumotacógrafo com uma peça adaptadora em formato de Y, uma peça bocal e um tudo de impedância (Figura 1). O gerador externo, constituído pelo alto-falante, é responsável por gerar um estímulo pulsátil por meio do adaptador em Y, o que resulta na resposta pulmonar que será registrada. Assim, existem dois sensores conectados ao pneumotacógrafo: o de débito e o de pressão bucal. Por meio destes, há o registro da ventilação espontânea do indivíduo avaliado. O pneumotacógrafo e o transdutor de pressão registram a atividade respiratória e o sinal de impulso forçado e, esses dados são processados e representados graficamente. ${ }^{2,6}$

\section{Parâmetros de avaliação}

Os parâmetros oscilométricos processados e suas características são expressos por meio de frequências de $5,10,15,20,25,30$ e 35 hertz. O IOS analisa a mecânica pulmonar sob outro ponto de vista $\mathrm{e}$, para isso, emite um modelo o qual reduz estruturas reais a componentes calculáveis. ${ }^{25}$ Sendo os parâmetros mais importantes analisados pelo sistema:

- impedância respiratória $(Z)$ : resistência respiratória complexa, que varia com a frequência sonora., ${ }^{2,3}$

- resistência respiratória $(R)$ : inclui a resistência proximal e distal das vias aéreas (central e periférica), do tecido pulmonar e parede torácica. Pode ser medida a $5 \mathrm{~Hz}$ (R5), que representa a resistência total das vias aéreas, ou a $20 \mathrm{~Hz}$, que representa a resistência central das vias aéreas. A diferença entre elas resulta na resistência periférica (R5-R20). 2,3

- reatância $(X)$ : é o componente reativo da impedância respiratória. Inclui força para mover a coluna aérea nas vias aéreas condutivas, definida como inertância (I), e também as propriedades elásticas periféricas expressas como a capacitância (C). A reatância capacitiva periférica (X5), mostra o aumento da negatividade tanto na obstrução quanto na restrição. ${ }^{2,3}$

- frequência de ressonância (Fres): é o ponto de encontro da linha gráfica da reatância com a linha zero $^{2,3}$, onde as resistências elásticas e inerciais se tornam iguais. Representa a frequência de oscilação do sistema na qual as forças inerciais e elásticas atingem a mesma grandeza, mas com sinais opostos e, portanto, se anulam. A observação do deslocamento desse ponto gráfico viabiliza a identificação de distúrbios obstrutivos. ${ }^{1}$

Os valores considerados pelo fabricante (Master Screen IOS, Erich Jaeger, Germany ${ }^{\circledR)}$ como significativos para análise clínica são: Z, R5, R20, X5 e Fres.

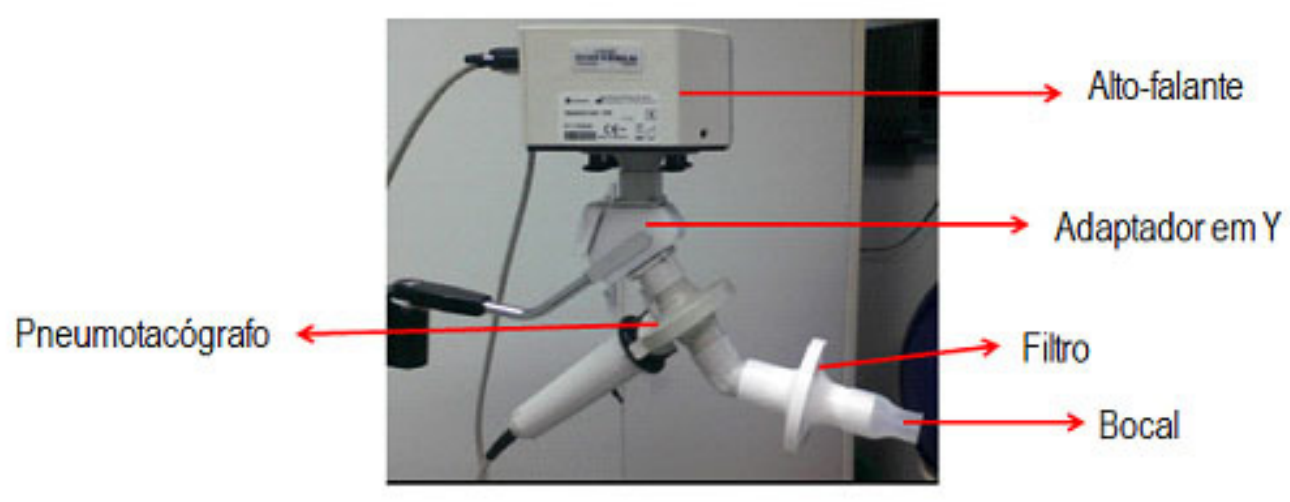

Figura 1: Equipamento de oscilometria de impulso. Fonte: elaborado pelos autores. Jaeger (Master Screen IOS, Erich Jaeger, Germany®). 


\section{Posicionamento e técnica de execução}

Geralmente o exame de oscilometria de impulso é realizado de acordo com as normas da American Thoracic Society (ATS). ${ }^{30}$ Após o aquecimento automático do sistema por cerca de 15 a 20 minutos é realizada a calibração do equipamento por meio de uma seringa de três litros e fornecido dados de condições ambientais (temperatura e umidade relativa do ar), através de um termo higrômetro digital (o exame não deve ser realizado se a temperatura do ambiente for inferior a $17^{\circ} \mathrm{C}$ e superior a $40^{\circ} \mathrm{C}$ ). Antes do início do exame, o indivíduo deve repousar por 10 a 15 minutos e receber instruções detalhadas sobre as etapas do procedimento de avaliação.

A técnica de execução é realizada enquanto o sujeito respira espontaneamente o ar ambiente por meio do tubo de um bocal. Enquanto isso, o gerador de impulso de pressão transmite breves impulsos de pressão ao trato respiratório, através de um adaptador em Y, um pneumotacógrafo e uma peça bocal.

$\mathrm{Na}$ maioria dos estudos encontrados na literatura, a técnica de execução segue os mesmos critérios. $\mathrm{O}$ indivíduo permanece na posição sentada durante o teste, é orientado a respirar calmamente através de um bocal (volume corrente e de forma espontânea), faz uso de um clipe nasal, e tem suas bochechas apoiadas pelas mãos do investigador para minimizar a perda de pressão oscilatória, decorrente da elevada complacência das mesmas e das vias aéreas superiores. ${ }^{31,32,33}$ (Figura 2). Somente, um estudo procedeu à coleta com as crianças em ortostatismo e fazendo uso de um guarda de língua. ${ }^{34}$

\section{Aceitabilidade e reprodutibilidade}

$\mathrm{Na}$ literatura, incluindo a faixa etária pediátrica, as medições do IOS consideradas como aceitáveis para a obtenção dos dados duram cerca de 15 a 20 segundos (s) de gravação. ${ }^{32,34,35,36}$ Alguns estudos recentes aceitam o período de $30 \mathrm{~s}^{37,12,16}$, entre 30 a $45 \mathrm{~s}^{37} \mathrm{e} 45 \mathrm{~s}^{38}$. Os autores Vink et al. ${ }^{39} \mathrm{e}$ Song et al. ${ }^{40,41}$ publicaram dados de até 60 s de gravação.

Durante esse período de registro, a criança necessita permanecer na postura adequada, sem falar, deglutir, chorar ou tossir durante as medições. ${ }^{32,33}$

Para análise de reprodutibilidade, as medidas de resistência oscilatória são repetidas três vezes e o coeficiente de variação deve ser menor que $0,15 .{ }^{34}$ Segundo Peirano ${ }^{33}$, um bom exame de resistência e impedância respiratória deve incluir a obtenção de três à cinco medidas aceitáveis, com um coeficiente de variação menor que 0,10 entre as medidas.

\section{Interpretação dos dados}

Conforme Peirano ${ }^{33}, \mathrm{Amra}^{5}$, Valle ${ }^{25}$, Cardoso e Ferreira ${ }^{1}$, Ferreira ${ }^{42}$ e informações contidas no manual do IOS (Master Screen IOS, Erich Jaeger, Germany $\left.{ }^{\circledR}\right)^{43}$ para a interpretação dos dados, o parâmetro que retrata o componente resistivo é a R. Esta reflete principalmente a perda de fricção que ocorre durante fluxo de ar nos brônquios, enquanto o não resistivo $(\mathrm{X})$ contempla a energia armazenada pelos componentes mais periféricos do sistema respiratório.

Um aumento na $\mathrm{R}$ com frequências menores (R5), sem nenhuma mudança na resistência a frequências mais altas (R20), associada a uma diminuição na reatância em frequências mais baixas (X5), representa um aumento na resistência de vias aéreas periféricas. Enquanto um aumento nas resistências R5 e R20, localizados na parte inferior e superior respectivamente, sem alteração da reatância sinalizam um aumento de resistência de vias aéreas centrais.

Para análise dos dados obtidos através do sistema, o equipamento gera gráficos e retas que representam o comportamento dos parâmetros de R, X, R5, R20 e Fres. Sendo um dos elementos gráficos consi-

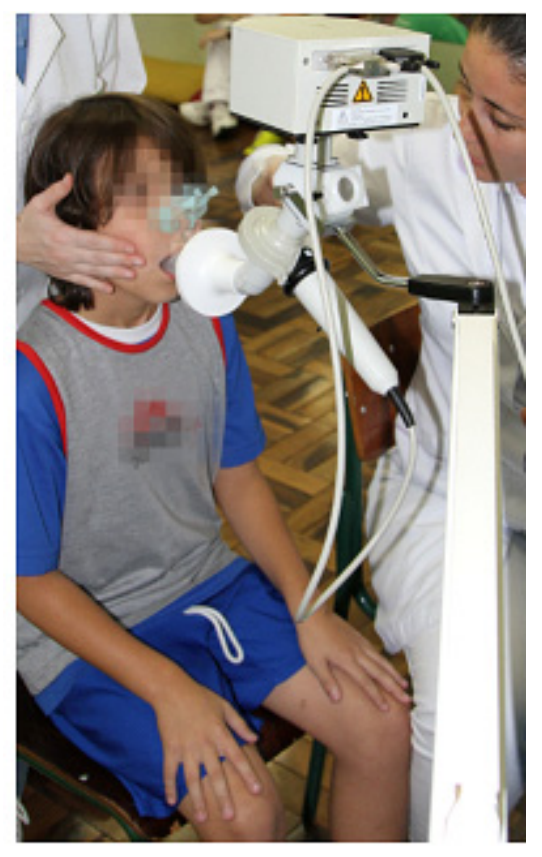

Figura 2: Representação do posicionamento adotado durante a execução do exame de oscilometria de impulso. Fonte: acervo autores. 
derado de maior importância é a reta de R, que contempla os valores de R5 e R20.

Observa-se que para cada situação clinica, existe um padrão de comportamento dos parâmetros captados. As características dos gráficos e cada uma das variáveis geralmente apresentam peculiaridades que serão apresentadas a seguir. Até o momento, não foram publicados traçados nem valores específicos relacionados à população pediátrica, sendo utilizados os mesmos dados indicados aos adultos.

\section{Representação gráfica e parâmetros para um indivíduo normal}

A figura 3 representa o gráfico de $R$, no qual o traçado é retilíneo e linear, constante e dentro da faixa de normalidade. Para que o exame seja considerado normal, os valores de R5 e R20 devem estar próximos do traçado do predito. Na análise do gráfico de $X$ (figura 3B), o individuo sem alteração apresenta tra- çado linear e ascendente, dentro do predito. $\mathrm{O}$ valor da frequência de ressonância não deve ultrapassar $10 \mathrm{~Hz}$. O gráfico demonstrado na figura 3C representa a diferença entre as resistências central (R20) e periférica (R5).

\section{Representação gráfica e parâmetros para um indivíduo com obstrução central}

Para que o exame indique uma obstrução central, os valores de R5 e R20 apresentam-se acima do predito, a resistência é representada na figura 4A, onde, a curva é retilínea e linear, porém dentro da faixa anormal. Na análise do gráfico de $X$ (figura 4B), o indivíduo com obstrução central apresenta um traçado linear, ascendente e dentro do normal. A Fres permanece dentro da normalidade $(10 \mathrm{~Hz})$. O gráfico demonstrado na figura 4C mostra que a resistência central é maior ou igual à resistência periférica (figura 4C).
A

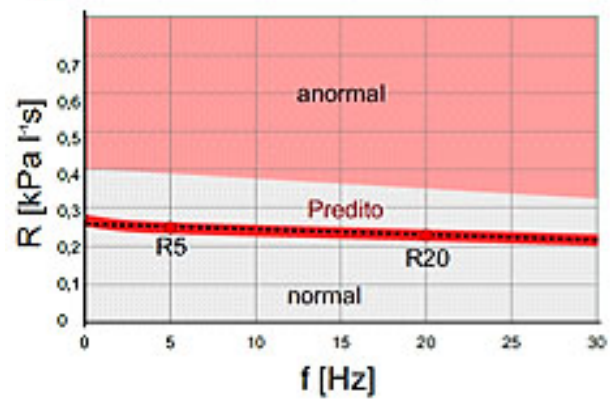

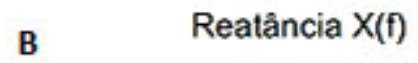

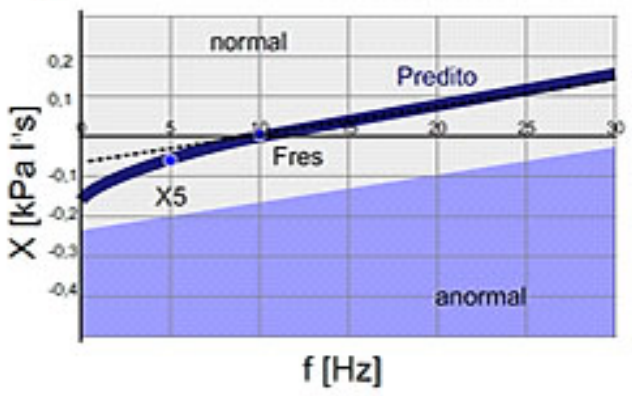

C

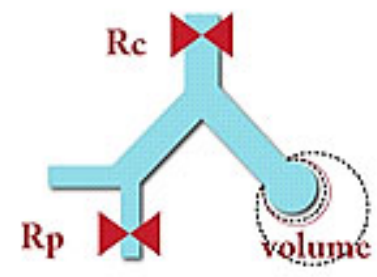

Figura 3: Representação gráfica e parâmetros para um indivíduo normal.

Legenda: R: resistência; Hz: Hertz; f: frequência; R5: resistência a 5Hz; R20: resistência a 20Hz; X reatância; X5: reatância a 5Hz; X20: reatância a $20 \mathrm{~Hz}$; Fres: frequência de ressonância; Rc: resistência central; Rp: resistência periférica. Baseado Manual Master Screen IOS, Erich Jaeger, Germany®
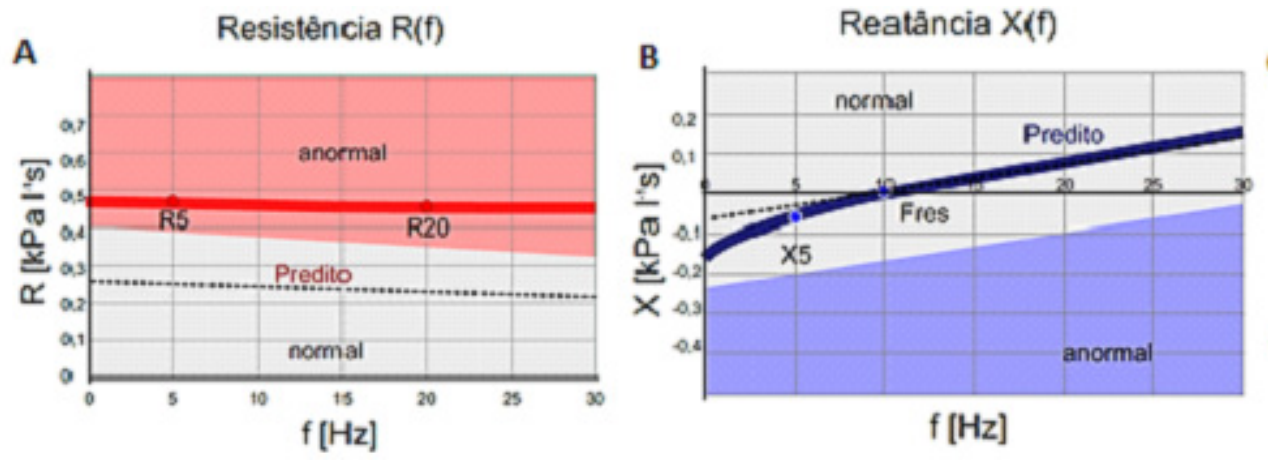

C

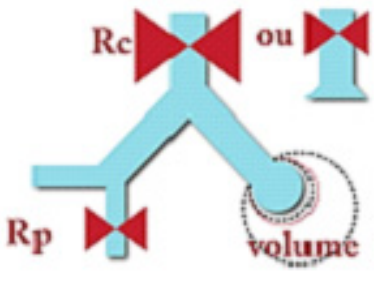

Figura 4: Representação gráfica e parâmetros para um indivíduo com obstrução central.

Legenda: R: resistência; Hz: Hertz; f: frequência; R5: resistência a 5Hz; R20: resistência a 20Hz; X reatância; X5: reatância a 5Hz; X20: reatância a $20 \mathrm{~Hz}$; Fres: frequência de ressonância; Rc: resistência central; Rp: resistência periférica. Baseado Manual Master Screen IOS, Erich Jaeger, Germany® 


\section{Representação gráfica e parâmetros para um} indivíduo com obstrução periférica

$\mathrm{O}$ gráfico de $R$, na figura $5 \mathrm{~A}$, mostra um traçado descendente, onde seu início está dentro da faixa anormal e termina dentro da normal. $\mathrm{Na}$ análise do gráfico de $X$, representada pela figura $5 \mathrm{~B}$, este indivíduo apresenta traçado ascendente, no qual o valor de X5 inicia com um valor muito abaixo da normalidade e o valor de Fres é deslocado para direita. Em indivíduos com quadro de obstrução periférica, o valor da resistência periférica é maior que a resistência central (figura 5C).

\section{Representação gráfica e parâmetros para um indivíduo com restrição pulmonar}

Nos casos de restrição pulmonar, observa-se um gráfico de $R$ linear e próximo do predito (figura 6A). $\mathrm{Na}$ análise do gráfico de $X$, na figura $6 \mathrm{~B}$, o traçado é ascendente com início na faixa anormal e término na normal, onde o X5 começa muito abaixo do normal e a Fres está deslocada para direita. Os valores de resistência periférica e central estão próximos (figura 6C).

\section{Representação gráfica e parâmetros para um} indivíduo com obstrução extratorácica

Em situações onde há a presença de obstrução extratorácica, o gráfico de $R$ apresenta um traçado dentro do espaço definido como anormal, onde R5 e R20 estão aumentados. Entre esses dois parâmetros identifica-se a presença de um pico no traçado (figura 7A). Na análise do gráfico de $X$ (figura 7B) o traçado inicia-se com o valor de X5 abaixo do normal, apresenta um platô e entra na normalidade. A Fres é discretamente deslocada para direita. Há presença de air-trapping no gráfico com "espículas". Nesse caso, não há relação entre a resistência central e periférica, portanto o gráfico representado pela figura $7 \mathrm{C}$, não deve ser interpretado.
A

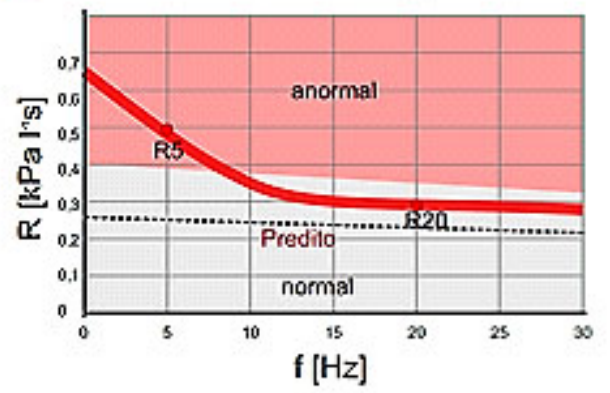

B

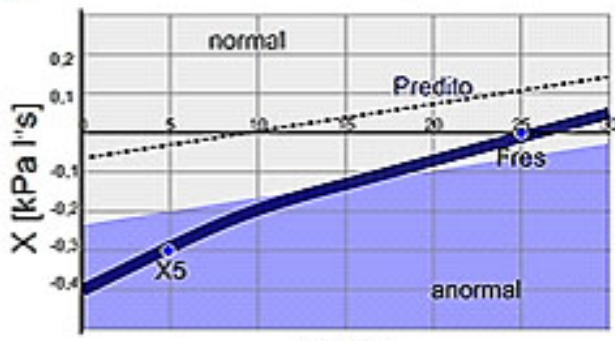

$f[H z]$
C

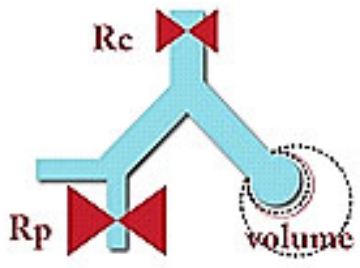

Figura 5: Representação gráfica e parâmetros para um indivíduo com obstrução periférica.

Legenda: R: resistência; Hz: Hertz; f: frequência; R5: resistência a $5 \mathrm{~Hz}$; R20: resistência a $20 \mathrm{~Hz}$; X reatância; X5: reatância a $5 \mathrm{~Hz}$; X20: reatância a 20Hz; Fres: frequência de ressonância; Rc: resistência central; Rp: resistência periférica. Baseado Manual Master Screen IOS, Erich Jaeger, Germany®

A

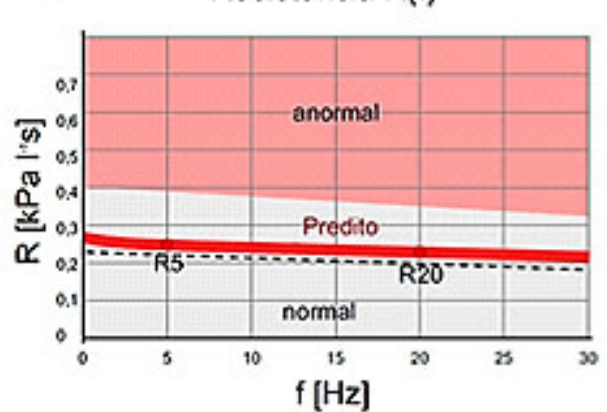

B

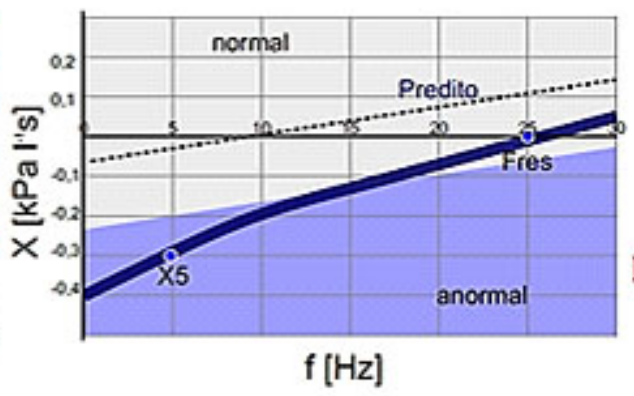

C

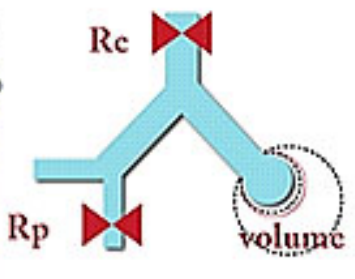

Figura 6: Representação gráfica e parâmetros para um indivíduo com restrição pulmonar

Legenda: R: resistência; Hz: Hertz; f: frequência; R5: resistência a $5 \mathrm{~Hz}$; R20: resistência a $20 \mathrm{~Hz}$; X reatância; X5: reatância a $5 \mathrm{~Hz}$; X20: reatância a $20 \mathrm{~Hz}$; Fres: frequência de ressonância; Rc: resistência central; Rp: resistência periférica. Baseado Manual Master Screen IOS, Erich Jaeger, Germany® 
A

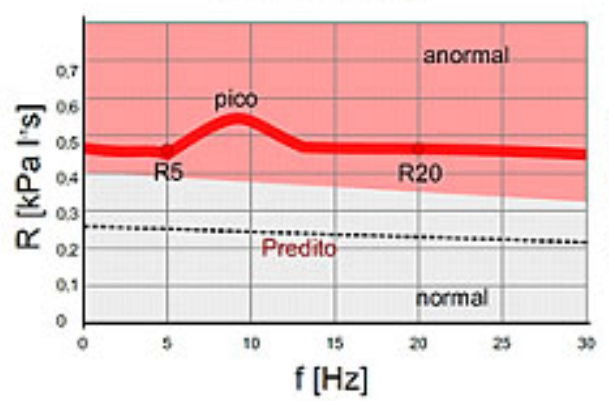

B

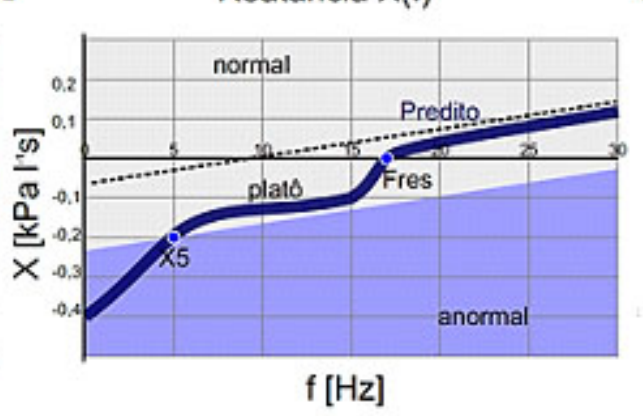

c

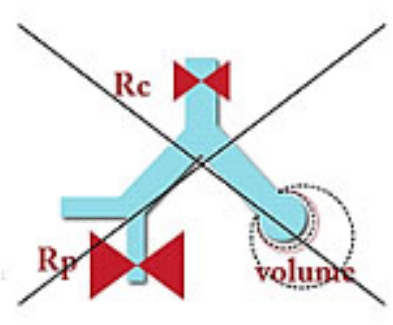

Figura 7: Representação gráfica e parâmetros para um indivíduo com obstrução extratorácica.

Legenda: R: resistência; Hz: Hertz; f: frequência; R5: resistência a 5Hz; R20: resistência a 20Hz; X reatância; X5: reatância a 5Hz; X20: reatância a $20 \mathrm{~Hz}$; Fres: frequência de ressonância; Rc: resistência central; Rp: resistência periférica. Baseado Manual Master Screen IOS, Erich Jaeger, Germany®

\section{IOS em pediatria}

Muitos métodos que objetivam verificar a obstrução das vias respiratórias que foram desenvolvidos para adultos, tem mostrado aplicabilidade em pediatria, sendo indicados tanto para crianças saudáveis, quanto para aquelas com diagnóstico de asma $\mathrm{ma}^{37,40,44-50}$ e fibrose cística. ${ }^{48}$

Nos últimos anos, a atenção tem se voltado para o uso do IOS na avaliação da reatividade das vias aéreas, em especial nas crianças asmáticas, com o objetivo de verificar a resposta broncodilatadora nesses pacientes. ${ }^{12,37,41,49-54}$

Nessa linha Marotta et al. ${ }^{12}$ verificaram a resposta ao broncodilatador em 73 crianças de 4 anos de idade propensas a serem asmáticas. Para tal, compararam os resultados obtidos através do IOS com dados espirométricos. Os participantes realizaram exames de IOS e espirometria antes e depois da administração de albuterol, identificando-se alterações de R5 e R10 somente nas crianças asmáticas. Diante da detecção de resposta ao broncodilatador com o uso do IOS, os autores consideraram a oscilometria de impulso como uma ferramenta útil para o diagnostico precoce da asma.

A mesma sensibilidade do IOS quanto a resposta brondilatadora foi verificada no estudo de Song et al. ${ }^{41}$, que comparou a utilidade do IOS e da espirometria em 77 crianças coreanas asmáticas e 55 controles saudáveis (idades entre 3 e 6 anos), antes e após a administração de broncodilatador. Em seus resultados, os autores evidenciaram que as crianças asmáticas apresentaram variações na R5, R10, R20 e R35 no IOS, em comparação às crianças hígidas, sendo
R5 a variável de maior representatividade quanto aos efeitos da medicação. A espirometria não apresentou alteração, mas alguns de seus parâmetros de volume expiratório forçado no primeiro segundo $\left(\mathrm{VEF}_{1}\right)$, capacidade vital forçada (CVF) e pico de fluxo expiratório (PFE) tiveram correlação significativa com dados do IOS (R10, R20 e R35).

Outra pesquisa de mesmo enfoque foi conduzida por Meraz et al. ${ }^{37}$, que constataram que os parâmetros do IOS diferiram consistentemente nas crianças asmáticas, em comparação as saudáveis, sendo capaz de discriminar distúrbios obstrutivos e não obstrutivos. O trabalho envolveu 26 crianças asmáticas analisadas pelo IOS por um ano, sem o uso do broncodilatador e, dois anos depois, foram examinadas antes e após o seu uso.

Nos fibrocísticos, as pesquisas são direcionadas para se observar as relações entre parâmetros da espirometria e do IOS, já que esta "dupla" parece se complementar na avaliação da resistência das vias aéreas ${ }^{19,55}$. Por essa característica, o IOS vem se estabelecendo progressivamente na literatura. ${ }^{2}$

Nessa linha, Moreau et al. ${ }^{32}$ avaliaram 15 indivíduos com fibrose cística entre 4 e 19 anos, por meio do IOS e da espirometria, considerando R5, X5, Z e Fres; $\mathrm{VEF}_{1}$ fluxo expiratório forçado entre 25 e $75 \%$ da CVF $\left(\mathrm{FEF}_{25 \%-75 \%}\right)$ e CVF, respectivamente. Após o acompanhamento desses pacientes, em 5 ocasiões isoladas e com intervalos variados, os autores observaram uma relação inversa entre os valores de R5, Z e Fres e os parâmetros espirométricos. Diante desses achados, constatou-se que o IOS não apresentou sensibilidade suficiente para detectar e acompanhar a obstrução brônquica de fibrocísticos. 
Em 2006, Díez et al. ${ }^{20}$ avaliaram o comportamento dos parâmetros espirométricos e oscilométricos de 39 fibrocísticos, e observaram correlações entre $\mathrm{VEF}_{1}$ e algumas variáveis oscilométricas $(\mathrm{Z}, \mathrm{R} 5$, X5). Para a ausência de correlação com a R, atribuiuse o fato da determinação desse parâmetro ser condicionado a resistência das vias aéreas centrais, portanto, de maior calibre, enquanto na fibrose cística a obstrução ocorre principalmente em vias aéreas mais periféricas. Reforçando essa explicação dos autores, as medidas de $\mathrm{X}$, que são determinadas principalmente pelo calibre das vias aéreas mais distais, apresentaram correlação significativa com os valores de $\mathrm{VEF}_{1}$

Um ponto relacionado aos achados expostos e que deve ser considerado em estudos com pneumopatas que apresentam instabilidade brônquica, é que durante a expiração forçada pode haver o colapso das vias aéreas e, desse modo, ocorrer um declínio do parâmetro espirométrico de $\mathrm{VEF}_{1}$. Em contrapartida, segundo Hellinckx et al. ${ }^{56}$, as medidas oscilométricas que envolvem a resistência das vias aéreas podem se manter perto da normalidade, pois este método envolve a respiração corrente.

Apesar da relevância dos estudos supracitados, o IOS é utilizado como ferramenta para identificação e acompanhamento de alterações do sistema respiratório decorrentes de disfunções, como asma e fibrose cística. No entanto, observa-se a escassez de estudos envolvendo crianças saudáveis, o que dificulta comparações e o estabelecimento de parâmetros de normalidade. ${ }^{40}$ Recentes e poucos são os estudos que discutem essa questão e, menos ainda, são as pesquisas destinadas a criação de valores de referência do IOS em pediatria.

\section{Valores de referência do IOS para faixa etá- ria pediátrica}

A geração de valores de referência em métodos de avaliação de diferentes populações norteiam pesquisas e viabilizam comparações. ${ }^{57,58,59}$ Nesse âmbito, a condução desse tipo de estudo envolvendo parâmetros do IOS, é relativamente recente.

Em pediatria até o momento são poucas as publicações sobre o tema em diferentes nacionalidades. Nos estudos identificados na corrente revisão $0^{5,34,35,43,58,60,61,62}$, os valores de referência foram obtidos pela análise de regressão linear múltipla e transformações logarítmicas e identificam a estatura como o melhor fator preditivo para os parâmetros oscilométricos. ${ }^{4,34,35,38,57,59,60}$ Estes trabalhos apresentam faixas etárias variadas, diferentes frequências de captação dos dados e divergência em seus tamanhos amostrais.

Dentre eles, o estudo de Frei et al..$^{34}$, analisou 222 crianças norte americanas, entre 3 e 10 anos. Os autores relacionaram os parâmetros do IOS com variáveis antropométricas (peso e estatura), idade e sexo, sendo avaliados os parâmetros em frequências de 5, $10,15,20,25$ e $35 \mathrm{~Hz}$, com um tempo de aquisição de 15 a 20 segundos.

Com o objetivo de apresentar valores abrangentes de determinantes da impedância do sistema respiratório, Dencker et al. ${ }^{35}$ realizaram uma pesquisa cujas medições do IOS foram realizadas num período de pelo menos 20 segundos, em frequências de 5 a $20 \mathrm{~Hz}$. Também foram analisadas as variáveis de peso, idade, área de superfície corporal (ASC) e sexo em 109 crianças finlandesas (entre 2 e 7 anos) e 252 suecas ( entre 7 e 11 anos).

Já o estudo de Ai et al. ${ }^{57}$, foi realizado com 549 crianças chinesas de 4 a 14 anos, divididas em 10 faixas etárias. Verificou-se que a impedância total e resistência das vias aéreas foram correlacionadas negativamente com a estatura e idade das crianças, sendo a estatura classificada em primeiro lugar e idade em segundo. No trabalho se discute o fato de que a aplicação dos valores previstos respeite a população correspondente na determinação da mecânica respiratória, uma vez que existem características diferentes em cada região e país.

Considerado o primeiro estudo sobre valores de referência para o IOS em crianças coreanas, Wee et al. ${ }^{61}$ determinaram tais valores com uma amostra de 92 crianças entre 7 e 12 anos. Da mesma forma, o trabalho de Nowowiejska et al. ${ }^{58}$ incluiu crianças e adolescentes poloneses entre 3 e 18 anos, e evidenciou a estatura como a variável de melhor correlação com os parâmetros oscilométricos. Os parâmetros do IOS foram R e X de 5, 10, 15, 20, 25 e 35 Hz, Fres e X, e o tempo de registro dos dados foi de 45 segundos.

Valores de referência do IOS para crianças e adolescentes iranianos também já foram publicados ${ }^{5}$, sendo destacado alto poder preditivo da idade e da estatura sobre os parâmetros oscilométricos considerados (R5, R15, R25, X5, X15 e X25). A amostra foi constituída por 509 indivíduos, sendo 265 meninos entre 6 e 19 anos, e 253 meninas entre 5 e 19 anos, em estudo que também analisou a idade, peso, índice de massa corporal (IMC) e ASC, de acordo com cada sexo. 
O estudo mais recente sobre valores de referência para o IOS é o de Park et al. ${ }^{60}$ e Lee et al. ${ }^{59} \mathrm{O}$ primeiro trata-se de uma pesquisa com 119 pré-escolares coreanos, com idades entre 3 e 6 anos, que teve como objetivo gerar valores de referência do sistema de impedância respiratória em frequências de 5 e 10 Hz. Para tal, foi aplicada análise de regressão linear múltipla entre a relação dos parâmetros de resistência e reatância, com dados antropométricos (estatura e peso) e idade. O estudo de Lee et al. ${ }^{59}$ avaliou 390 coreanas de 3 a 7 anos e, assim como nas outra pesquisa, a variável estatura foi a que teve maior correlação com os dados do IOS. Os autores discutem sobre a utilização dos valores de referência para a avaliação de crianças asmáticas, sugerindo que o valor previsto de R5 está relacionado como valor de corte para a obstrução das vias aéreas, o que pode indicar uma obstrução aérea com significante fator de risco para o diagnóstico da asma.

\section{Relação entre IOS e outros métodos de ava- liação do sistema respiratório}

Como apresentado nos estudos envolvendo o IOS na prática clinica, alguns trabalhos comparam e relacionam o IOS com outros métodos de avaliação do sistema respiratório, especialmente sua associação com parâmetros espirométricos na população pediátrica. ${ }^{39,12,14,38,41,62}$

Vem sendo constatada uma forte correlação entre algumas medidas do IOS e o $\mathrm{VEF}_{1}$, como nos estudos de Nowowiejska et al. ${ }^{38}$ e de Kalahoff et al. ${ }^{62}$ Vink et al. ${ }^{39}$ no qual, observaram que mudanças significativas na obstrução do fluxo aéreo foram identificadas por meio de correlações entre os valores de $\mathrm{R}$ e $\mathrm{X}$ com o $\mathrm{VEF}_{1}$.

Nos resultados obtidos por Song et al..$^{41}$ as medidas de $\mathrm{VEF}_{1}$ e PFE apresentaram correlação significativa com Z e R a 5, 10, 20 e $35 \mathrm{~Hz}$, em asmáticos atópicos e em crianças saudáveis, sendo que as medidas de CVF também mostraram correlação com Z e com R em $10 \mathrm{~Hz}$. A medida de FEF $25 \%-75 \%$ também teve correlação com parâmetro oscilométrico de $\mathrm{X}^{18}$, parecendo complementar informações fornecidas pelo $\mathrm{VEF}_{1}$.

Além da espirometria, algumas pesquisas comparam o IOS com outros métodos de avaliação do sistema respiratório, para avaliação de pneumopatias pediátricas. Nessa linha, Olagúibel et al. ${ }^{63}$ avaliaram os efeitos pulmonares da resposta broncodilatadora antes e após a aplicação do sabultamol em 33 crian- ças asmáticas de 3 a 6 anos de idade. O IOS foi bem aceito pelas crianças e foi capaz de fornecer dados reprodutíveis e sensíveis da função pulmonar, sendo os valores de resistência em baixa frequência (R5) correlacionados com os valores de espirometria e pletismografia.

Também em pediatria, Klug e Bisgaard ${ }^{64}$ buscaram avaliar três testes pulmonares em 20 crianças asmáticas estáveis de 2 a 4 anos, a oscilometria de impulso, a técnica do interruptor (Rint) e medida de tensão transcutânea de oxigênio $\left(\mathrm{Ptc}, \mathrm{O}_{2}\right)$ concomitantemente com as mensurações de resistência específica das vias aéreas, antes e após a aplicação de metacolina. Os autores relatam que todas as técnicas descritas conseguiram refletir as mudanças em curto prazo na função respiratória, com a principal vantagem de não serem invasivas e serem menos dependentes da cooperação ativa dos indivíduos, sendo alternativas bem adequadas à população pediátrica. Esses mesmos autores, no ano de $1995^{65}$, também relacionaram as medidas do IOS, Rint e Ptc, $\mathrm{O}_{2}$ com medidas de $\mathrm{VEF}_{1}$ por pletismografia de corpo inteiro e espirometria, em 21 crianças entre 4 e 6 anos, com suspeita de asma. Observou-se que as medidas obtidas pelo IOS, Rint e Ptc, $\mathrm{O}_{2}$ modificam-se paralelamente com as mensurações de resistência das vias aéreas e $\mathrm{VEF}_{1}$, e apresentam sensibilidades comparáveis para detectar a resposta broncodilatadora da metacolina.

Os estudos acima relatados incluíram a população asmática e tiveram como principal objetivo verificar qual método seria mais sensível para detectar mudanças respiratórias mediante administração de medicações inalatórias, constatando que, tanto o IOS como a pletismografia corporal, e os outros métodos descritos, podem ser utilizados para esse fim. Com base nesses trabalhos, as medidas obtidas pelo IOS vêm ganhando espaço para distinguir efeitos antes e após a administração de medicações broncodilatadoras, sendo mais um instrumento além da espirometria.

Relações entre o IOS e a pletismografia também foram investigadas por Tomalak et al. ${ }^{7}$ em uma pesquisa que incluiu 334 indivíduos, com idades entre 5 e 18 anos, apresentando disfunções respiratórias (asma, doenças alérgicas, fibrose cística, bronquiectasias e fibrose pulmonar) fora do quadro de exacerbação. As relações entre os resultados das medições da pletismografia e das resistências a 5, 20 e $35 \mathrm{~Hz}$ do IOS foram analisadas. Todas as três resistências apresentaram correlações positivas com a pletismografia, sendo a mais forte observada em R5. Os auto- 
res concluíram que IOS pode ser útil no diagnóstico de doenças respiratórias obstrutivas, além de ser um método mais simples, quando comparado à pletismografia corporal.

Segundo Peirano ${ }^{33}$, embora os primeiros estudos sobre métodos de oscilação forçada datem de mais de 50 anos, parece que a compreensão e a utilização das medições das resistências respiratórias, em especial do IOS é ainda pouco frequente e carente de elucidações. Sua relação com outros instrumentos de avaliação, principalmente os já estabelecidos na comunidade científica, merece ser evidenciada para que se esclareça ainda mais sua indicação.

\section{Conclusão}

A presente revisão evidenciou considerável número de materiais referente ao equipamento de oscilometria de impulso, seus parâmetros de avaliação, considerações sobre a técnica de execução e o posicionamento do paciente, critérios de aceitabilidade e reprodutibilidade do exame, bem como interpretação dos dados obtidos.

São muitas as publicações voltadas para o estudo dessa ferramenta de análise da mecânica respi- ratória, no entanto, informações referentes ao equipamento, detalhes técnicos e os parâmetros de avaliação do sistema ainda são restritas ao próprio manual do fabricante. Sobre o posicionamento do paciente e a técnica de execução do exame ainda encontram-se divergências entre os autores, principalmente em relação ao tempo de aquisição das medidas, assim como nos critérios de aceitabilidade e reprodutibilidade. As pesquisas atentam para importância do conhecimento dos parâmetros do IOS nas diferentes situações clínicas para que haja uma adequada interpretação dos dados e gráficos.

Na pediatria, recentes publicações têm apresentado valores de referência de diferentes populações e a relação do sistema com outros métodos de avaliação respiratória. Nessa linha, a maioria dos estudos analisa qual é o instrumento mais sensível para detectar mudanças respiratórias e a resposta diante da administração de medicações inalatórias.

Consensual entre os autores do material teórico analisado é que o IOS é um recurso relativamente novo, cuja indicação na faixa etária pediátrica tem sido crescente, mas que ainda carece investigações, tanto técnicas quanto relativas à sua aplicabilidade clínica.

\begin{abstract}
Introduction: impulse oscillometry (IOS) is considered a method of evaluation of respiratory mechanics, and being non-invasive, has been increasingly mentioned in the care and research of pediatric patients. Studies have considered the interest of determining reference values and check the IOS relationship with other methods of respiratory assessment. Objective: to present an update of the literature regarding the system, technical criteria and interpretation of data, as well as the relationship of the system with other methods of respiratory assessment and its clinical applicability. Method: literature review with consultation in the databases MEDLINE / PubMed and Ebsco, using the keywords: "impulse oscillometry", "reference values" and "child". It was considered all material with information about the equipment, their indications and application as an evaluation tool in different clinical situations. Results: 45 documents were identified, (41 trials and 4 reviews), supplemented with 20 papers (1 guideline, 1 manual on equipment and 18 articles on the subject). This material was characterized by approaching the equipment, its parameters evaluation, performance technique and positioning of the patient during the examination, criteria for acceptability and reproducibility, and interpretation of the data obtained. There are several publications of this tool in the evaluation of respiratory mechanics disorders such as asthma and cystic fibrosis, and making on reference values in healthy children. Conclusion: IOS is a relatively new feature, whose indication in pediatrics has been increasing, but still required investigations, both technical and clinical investigations.
\end{abstract}

Key-words: Oscillometry. Reference Values. Child. Respiratory Function Tests. 


\section{Referências Bibliográficas}

1. Cardoso AP, Ferreira JMR. Oscilometria de impulso. Novo método de avaliação da função respiratória. Rev Port Pneumol. 1998; 2: 175-205.

2. Moreira MAF. A contribuição da oscilometria de impulso na obstrução de vias aéreas. [Tese Doutorado Pneumologia]. Faculdade de Medicina da Universidade Federal do Rio Grande do Sul (UFRGS), Porto Alegre, 2005.

3. Meraz E, Nazeran H, Goldman MD, Nava P, Diong B. Impulse oscillometric features of lung function: towards computer-aided classification of respiratory diseases in children. Conf Proc IEEE Eng Med Biol Soc. 2008; 2443-6.

4. Newbury W, Crockett A, Newbury J.A pilot study to evaluate Australian predictive equations for the impulse oscillometry system. Respirol. 2008; 13: 1070-5.

5. Amra B, Soltaninejad F, Golshan M. Respiratory resistance by impulse oscillometry in healthy Iranian children aged 519 years. Iran J Allergy Asthma Immunol. 2008; 7: 25-9.

6. Smith HJ, Reinhold P, Goldman MD. Forced oscillation technique and impulse oscillometry. Eur Respir Mon. 2005; 31:72105.

7. Tomalak W, Radli?ki J, Pawlik J, Latawier W, Pogorzelski A. Impulse oscillometry vs. pletismograpgy in assessing respiratory resistance in children. Pediatr Pulmonol. 2006; 41: 50-4.

8. Al-Mutairi SS, Sharma PN, Al-Alawi A, AI-Deen JS. Impulse oscillometry: an alternative modality to the conventional pulmonary function test to categorise obstructive pulmonary disorders. Clin Exp Med. 2007; 7: 56-64.

9. Klug B. The impulse oscillation technique applied for measurements of respiratory function in young children. Pediatr Pulmonol. Suppl 1997; 16:240-1.

10. Lum S. Lung function in preschool children: applications in clinical and epidemiological research. Paediatr Respir Rev. 2006; 7: 30-2.

11. Goldman MD, Carter R, Klein R, Fritz G, Carter B, Pachucki $P$. Within- and between-day variability of respiratory impedance, using impulse oscillometry in adolescent asthmatics. Pediatr Pulmonol. 2002; 34: 312-9.

12. Marotta A, Klinnert MD, Price MR, Larsen GL, Liu A. H. Impulse oscillometry provides an effective measure of lung dysfunction in 4-year-old children at risk for persistent Asthma. J Allergy Clin Immunol. 2003; 112:317-22.

13. Horsman TA, Duke RK, Davenport PW. Airway Response to Mannitol Challenge in Asthmatic Children Using Impulse Oscillometry. J Asthma. 2009; 46: 600-3.

14. Larsen GL, Morgan W, Heldt GP, Mauger DT, Boehmer SJ, Chinchilli VM, et al. Impulse oscillometry versus spirometry in a long-term study of controller therapy for pediatric asthma. J Allergy Clin Immunol 2008; 123: 861-7.

15. Frey U. Forced oscillation technique in infants and Young children. Paediatr Respir Rev. 2005; 6: 246-54.

16. Shi Y, AlediaAS, Tatavoosian AV, Vijayalakshmi S, Galant SP, George SC. Relating small airways to asthma control by using impulse oscillometry in children. J Allergy Clin Immunol. 2012; 129: 671-8.

17. Komarow HD, Skinner J, Young M, Gaskins D, Nelson C, Gergen PJ, et al. A study of the use of impulse oscillometry in the evaluation of children with asthma: analysis of lung parameters, order effect, and utility compared with spirometry. Pediatr Pulmonol. 2012; 47:18-26.

18. Shin YH, Yoon JW, Choi SH, Baek JH, Kim HY, Jee HM, et al.
Use of impulse oscillometry system in assessment of asthma severity for preschool children. J Asthma. 2013; 50: 198-203.

19. Minarowska A, Kaczmarski M, Minarowki L, Mrugacz M. The assessment of respiratory status in patients with cystic fibrosis: bodypletysmography or impulse oscillometry? J Cyst Fibros. 2005; 4: 59-73.

20. Díez JM, Villa Asensi JR, Angelo AV. Resistencias por oscilometria. Comparación de sucomportamiento em pacientes con asma y fibrosisquística. Rev Clin Esp. 2006; 206: 95-7.

21. Moreau L, Crenesse D, Berthier F, Albertini M. Relationship between impulse oscillometry and spirometric indices in cystic fibrosis children. Acta Paediatr. 2009; 98: 1019-23.

22. Arshi S, Nabavi M, Babaie D, Bahrami A, Ghalehbaghi B. Exercise induced changes in spirometry and impulse oscillometry measurements in persistent allergic rhinitis. Iran $\mathrm{J}$ Allergy Asthma Immunol. 2012; 11:259-66.

23. Komarow HD, Myles IA, Uzzaman A, Metcalfe D. Impulse oscillometry in the evaluation of diseases of the airways in children. Ann Allergy Asthma Immunol. 2011; 106:191-9.

24. Cavalcanti JV, Jansen JM, Melo PL. Avaliação das alterações mecânicas ao longo do ciclo respiratório: metodologia e aplicação em asmáticos. IFMBE Proceedings 2007; 18: 602-606.

25. Valle ELT. Resistência das vias aéreas: técnica da oscilação forçada. J Pneumol. 2002; 28:151-5.

26. Doing B, Rajagiri A, Goldman M, Nazeran H. The augmented RIC model of the human respiratory system. Med Biol Eng Comput. 2009; 47: 385-404.

27. Meraz EG. Homer N, Ramos C, Rodriguez L, Madrigal LR; Castillo NG. Impulse oscillometric features and respiratory system models track small airway function in children. Practical Applications on Biomedical Engineering [DOI:10,5772/52579]

28. Goldman MD, Nazeran H, Ramos C, Toon E, Oates K, Bilton $D$, et al. Electrical circuit models of the human respiratory system reflect small airway impairment measure by impulse oscillation (IOS). Conf Proc IEEE Eng Med Biol Soc. 2010; 2010: 2467-72.

29. Diong B, Nazeran H, Nava P. Modeling human respiratory impedance. Comparing the best method with the least estimation errors. IEEE Eng Med Biol Mag. 2007; 48-55.

30. Beydon N, Davis SD, Lombardi E, Allen J, Arets HG, Aurora P, et al. An official American Thoracic Society/European Respiratory Society statement: pulmonary function testing in preschool children. Am J Respir Crit Care Med. 2007; 175: 1304-45.

31. Ducharme FM, Davis GM, Ducharme GR. Pediatric reference values for respiratory resistance measured by forced oscillation. Chest. 1998; 113: 1322-8.

32. Malmberg LP, Pelkonen A, Poussa T, Pohianpalo A, Haahtela $\mathrm{T}$, Turpeinen M. Determinants of respiratory system input impedance and bronchodilator response in healthy Finnish preschool children. Clin Physiol Funct Imaging. 2002; 22: 6471.

33. Peirano, K. R. M. Oscilometría de impulso (IOS) em niños. Neumol Pediatr. 2010; 5: 89-95.

34. Frei J, Jutla J, Kramer G, Hatzakis GE, Ducharme FM, Davis GM. Impulse oscillometry : Reference values in children 100 to $150 \mathrm{~cm}$ in height and 3 to 10 years of age. Chest. 2005; 128: 1266-73.

35. Dencker M, Malmberg LP, Valind S, Thorsson O, Karlsson MK, Pelkonen A, et al. Reference values for respiratory system impedance by using impulse oscillometry in children aged 211 years. Clin Physiol Funct Imaging. 2006; 26: 247-50. 
36. Linares PM, Concha IM, Meter RP. Correlación entre laespirometría y laresistencia y reactanciarespiratoria medida por oscilometría de impulso em niños asmáticos. Rev Chil Enferm Respir. 2002; 18: 90-8.

37. Meraz EG, Nazeran H, Ramos CD, Nava P, Diong B, Goldman MD. Analysis of impulse oscillometric measures of lung function and respiratory system model parameters in small airway-impaired and healthy children over a 2-year period. Biomed Eng Online. 2011; 10:1-21.

38. Nowowiejska B, Tomalak, W, Radli?ski J, Siergiejko G, Latawiec W, KACZMARSKI M. Transient reference values for impulse oscillometry for children aged 3-18 years. Pediatr Pulmonol. 2008; 43: 1193-7.

39. Vink GR, Arets HGM, Laag J, Cornelis K. E. Impulse oscillometry: a measure for airway obstruction. Pediatr Pulmonol. 2003; 35: 214-9.

40. Song TW, Kim KW, Kim ES, Park J, Sohn MH, Kim K. Utility of impulse oscillometry in Young children with asthma. Pediatr Allergy Immunol. 2008; 19: 763-8.

41. Song TW, Kim KW, Kim ES, Kim KE, Sohn, MH. Correlation between spirometry and impulse oscillometry in children with asthma. Acta Paediatr. 2008; 97: 51-4.

42. Ferreira JMR, Cardoso AP, Rodrigues F, Sá R. Artigo de revisão: Provas de função pulmonar - controlo de qualidade $\left(2^{\mathrm{a}}\right.$ parte). Rev Port Pneumol. 2002; 1: 25-57.

43. Manual IOS- Jaeger Impulse Oscillometry. Measurement Program IOS basic1e. cap. 15, p. 1-28. Versão 5.2 .

44. Shi Y, Aledia AS, Galant SP, George SC. Peripheral airway impairment measured by oscillometry predicts loss of asthma control in children. J Allergy Clin Immunol. 2013; 131:71823.

45. Shin YH, Jang SJ, Yoon JW, Jee HM, Choi SH, Yum HY, Han MY. Oscillometric and spirometric bronchodilator response in preschool children with and without asthma. Can Respir J. 2012; 19: 273-7.

46. Galant SP, Nickerson B. Lung function measurement in the assessment of childhood asthma: recent important developments. Curr Opin Allergy Clin Immunol. 2010; 10: 149-154.

47. Xiang L, Liu SY, Zhang Q, Li Z. Comparison of pulmonary function of asthma children determined with impulse oscillometry and maximal expiratory flow-volume curve. Chinese J Pediatr 2003; 41:52-3.

48. Cogswell JJ. Forced oscillation technique for determination of resistance to breathing in children. Arch Dis Child. 1973; 48: $259-66$

49. Ortiz G, Menendez R. The effects of inhaled albuterol and salmeterol in 2- to 5-year-old asthmatic children as measured by impulse oscillometry. J Asthma. 2002; 39: 531-6.

50. Kim HY, Shin YH, Jung W, Jee HM, Park HW, Han MY. Resistance and reactance in oscillation lung function reflect basal lung function and bronchial hyperresponsiveness respectively. Respirol. 2009; 14:1035-1041.

51. Galant SP, Morphew T, Newcomb RL, Hioe K, Guijon O, Liao $\mathrm{O}$. The relationship of the bronchodilator response phenotype to poor asthma control in children with normal spirometry. J Pediatr. 2011; 158: 953-599.
52. Jee HM, Kwak JH, Jung W, Han MY. Useful parameters of bronchial hyperresponsiveness measured with an impulse oscillation technique in preschool children. J Asthma. 2010; 47: 227-232.

53. Bailly C, Crenesse D, Albertini M. Evaluation of impulse oscillometry during bronchial challenge testing in children. Pediatr Pulmonol. 2011; 46:1209-14.

54. Shulze J, Smith HJ, Fuchs J, Herrmann E, Dressler M, Rose $M A$, et al. Methacholine challenge in young children as evaluated by spirometry and impulse oscillometry. Respir Med. 2012; 106: 627-34.

55. Villa Asensi JR, Díez JM, Vecchi A, Posadas AS, Rodríguez MAN, González AS. Assessment of lung function using forced impulse oscillometry in cystic fibrosis patients. Arch Bronconeumol. 1998; 34: 520-4.

56. Hellinckx J, De Boeck, Demedts M. Evaluation of impulse oscillation system: comparison with forced oscillation technique and body plethysmography. Eur Respir J. 2001; 18:56470 .

57. Ai T, Luo RH, Wang CL, Yang YJ, Bao YF, Liao HL, et al. Determination of the lung function by impulse oscillometry in 549 healthy children in Chengdu area. Chinese Journal of Pediatrics 2007; 45:742-5.

58. Serradilla JMR, Aznar IC, Juan GMJ, Andeu LJA. Alteraciones em la funciónpulmonaryenel período posnatal y preescolar. An Pediatr. 2003; 58: 59-68.

59- Lee, JY, Seo JH, Kim HY, Jung YH, Kwon JW, Kim BJ, et al. Reference values of impulse oscillometry and its utility in the diagnosis of asthma in young Korean children. J Asthma. 2012; 49: 811-16.

60- Park JH, Yoon JW, Shin YH, Jee HM, Wee YS, Chang SJ, et al. Reference values for respiratory system impedance using impulse oscillometry in healthy preschool children. Korean $\mathrm{J}$ Pediatr 2011; 54: 64-8.

61. Wee YS, Kim HY, Jung DW, Park HW, Shin YHS, Han MY. Reference values for respiratory impedance using impulse oscillometry in school-aged children in Korea. Korean $\mathrm{J}$ Pediatr 2007; 50: 862-7.

62. Kalhoff H, Breidenbach $\mathrm{R}$, Smith H, Marek W. Impulse oscillometry in preschool children and association with body mass index. Respirol. 2011; 16: 174-9.

63- Olaguíbel JM, Alvarez-Puebla MJ, Anda M, Gómez B, García $\mathrm{BE}$, Tabar Al, et al. Comparative analysis of the bronchodilator response measured by impulse oscillometry (IOS), spirometry and body plethysmography in asthmatic children. $J$ Investig Allergol Clin Immunol. 2005;15:102-6.

64. Klug $B$, Bisgaard $H$. Measurement of lung function in awake 2-4-year-old asthmatic children during methacholine challenge and acute asthma: a comparison of the impulse oscillation technique, the interrupter technique, and transcutaneous measurement of oxygen versus whole-body plethysmography. Pediatr Pulmonol. 1996; 21: 290-300.62.

65. Bisgaard $\mathrm{H}$, Klug $\mathrm{B}$. Lung function measurement in awake young children. Eur Respir J. 1995; 8: 2067-2075. 